\title{
Polinômios Ortogonais de Legendre em Várias Variáveis
}

\author{
Mariana Aparecida Delfino de Souza \\ Depto de Matemática Aplicada, IBILCE, UNESP, \\ 15054-000, São José do Rio Preto, SP \\ E-mail: maridelfino6@yahoo.com.br, \\ Cleonice Fátima Bracciali \\ Depto de Matemática Aplicada, IBILCE, UNESP, \\ 15054-000, São José do Rio Preto, SP \\ E-mail: cleonice@ibilce.unesp.br.
}

\section{RESUMO}

Os polinômios ortogonais em uma variável são ferramentas importantes na solução de diversos tipos de problemas e sua teoria contribui nos estudos relacionados à estabilidade numérica, equações diferenciais, frações contínuas, teoria da aproximação, entre outros. A teoria desses polinômios é amplamente estudada, com muitos trabalhos publicados na área, como o livro de T. S. Chihara [2].

Em várias variáveis, os estudos desses polinômios têm-se difundido com maior intensidade nas últimas décadas. Segundo C. F. Dunkl e Y. Xu em [3], o primeiro trabalho nessa área é o livro [1] "Fonctions Hypergéométriques et Hypersphériques - Polynomes D’Hermite" de P. Appell e J. Kampé de Feriét, de 1926, que foi tomado como base neste trabalho.

De acordo com [3], os poucos livros dedicados à teoria geral dos polinômios ortogonais em várias variáveis têm como ênfase o tipo clássico desses polinômios, ou seja, as famílias de polinômios cujas funções peso têm como domínio as regiões regulares: o quadrado, o simplex, a bola em $\mathbb{R}^{n}$ ou o próprio $\mathbb{R}^{n}$.

Aqui, apresentamos como os polinômios de Legendre em $n$ variáveis, de grau $m_{1}+\cdots+m_{n}$, denotados por $V_{m_{1}, \ldots, m_{n}}\left(x_{1}, \ldots, x_{n}\right)$, que são ortogonais na região da bola unitária, e algumas de suas propriedades, podem ser obtidas através da extensão de alguns de seus conceitos e propriedades destes polinômios já conhecidas em uma variável. Um exemplo desta extensão é a função hipergeométrica, que em uma variável é dada, para $V_{m}(x)$, por

$$
V_{m}(x)=2^{m}\left(\frac{1}{2}\right)_{m} \frac{x^{m}}{m !} F\left(\frac{-m}{2}, \frac{1-m}{2} ; \frac{1}{2}-m ; \frac{1}{x^{2}}\right),
$$

com

$$
F(a, b ; c ; x)={ }_{2} F_{1}\left(\begin{array}{c}
a, b \\
c
\end{array} ; x\right)=\sum_{j=0}^{\infty} \frac{\left(a_{1}\right)_{j}\left(a_{2}\right)_{j}}{\left(b_{1}\right)_{j} j !} x^{j},
$$

$\mathrm{e}(a)_{n}=a(a+1)(a+2) \cdots(a+n-1), n=1,2,3, \ldots \mathrm{e}(a)_{0}=1$.

Em várias variáveis, temos

$$
\begin{aligned}
V_{m_{1}, \ldots, m_{n}}\left(x_{1}, \ldots, x_{n}\right)= & 2^{\mu}\left(\frac{n}{2}\right)_{\mu} \frac{x_{1}^{m_{1}}}{m_{1} !} \cdots \frac{x_{n}^{m_{n}}}{m_{n} !} \\
& \times F_{B}\left(-\frac{m_{1}}{2}, \ldots,-\frac{m_{n}}{2}, \frac{1-m_{1}}{2}, \ldots, \frac{1-m_{n}}{2} ;-\frac{n}{2}-\mu+1 ; \frac{1}{x_{1}^{2}}, \ldots, \frac{1}{x_{n}^{2}}\right),
\end{aligned}
$$


onde

$$
F_{B}\left(\alpha_{1}, \ldots, \alpha_{n}, \beta_{1}, \ldots, \beta_{n} ; \gamma ; x_{1}, \ldots, x_{n}\right)=\sum_{j_{i}=0}^{\infty} \frac{\left(\alpha_{1}\right)_{j_{1}}\left(\beta_{1}\right)_{j_{1}} \ldots\left(\alpha_{n}\right)_{j_{n}}\left(\beta_{n}\right)_{j_{n}}}{(\gamma)_{j} j_{1} ! \ldots j_{n} !} x_{1}^{j_{1}} \ldots x_{n}^{j_{n}}
$$

$\operatorname{com} j=j_{1}+\ldots+j_{n}$.

Além da função geratriz e da relação de ortogonalidade, a analogia da extensão de uma para várias variáveis dos Polinômios de Legendre também pode ser observada na sequência lógica da demonstração das funções hipergeométricas, dadas anteriormente.

Palavras-chave: Polinômios ortogonais, Polinômios ortogonais de Legendre, Polinômios ortogonais em várias variáveis

\section{Referências}

[1] P. Appell, J. Kampé de Feriét, "Fonctions Hypergéométriques et Hypersphériques - Polynomes D’Hermite", Ed. Gauthuer-Villars et C ${ }^{i e}$, Paris, 1926.

[2] T. S. Chihara, "An Introduction to Orthogonal Polynomials", Serie Mathematics and its Applications v. 13, Gordon and Breach, New York, 1978.

[3] C. F. Dunkl, Y. Xu, “Orthogonal Polynomials of Several Variables”, Cambridge University Press, Cambridge, 2001.

[4] R. Koekoek, P. A. Lesky, R. F. Swarttouw, "Hypergeometric Orthogonal Polynomials and Their q-Analogues", Springer-Verlag, Berlin, 2010. 